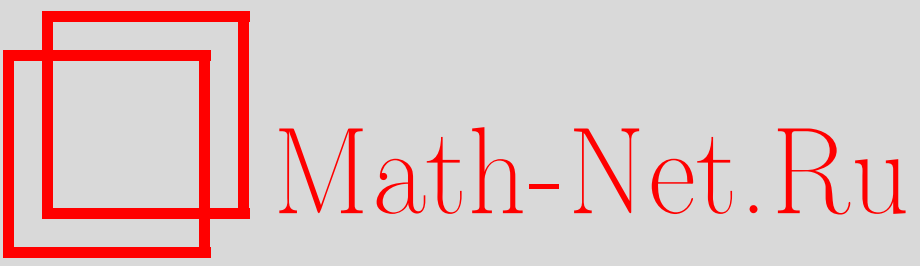

В. М. Бухштабер, Д. В. Лейкин, Трилинейные функциональные уравнения, УМH, 2005, том 60, выпуск 2, 151-152

DOI: https://doi.org/10.4213/rm1410

Использование Общероссийского математического портала Math-Net.Ru подразумевает, что вы прочитали и согласны с пользовательским соглашением

http://www.mathnet.ru/rus/agreement

Параметры загрузки:

IP : 3.85 .183 .62

26 апреля 2023 г., 12:32:51 


\title{
ТРИЛИНЕЙНЫЕ ФУНКЦИОНАЛЬНЫЕ УРАВНЕНИЯ
}

\author{
В. М. БУХШТАБЕР, Д. В. ЛЕЙКИН
}

Пусть $u_{1}, \ldots, u_{k-1}, z \in \mathbb{C}^{g}$ и $D$ - линейньй дифференциальньй оператор по $z$ с постоянными коэффициентами. Оператору $D$ ставится в соответствие $k$-линейньй оператор $\mathscr{D}$ по формуле

$\mathscr{D}\left(f_{1}, \ldots, f_{k}\right)\left(u_{1}, \ldots, u_{k-1}\right)=\left.D\left[f_{1}\left(u_{1}+z\right) \cdots f_{k-1}\left(u_{k-1}+z\right) f_{k}\left(\left(u_{1}+\cdots+u_{k-1}\right)-z\right)\right]\right|_{z=0}$.

Уравнение вида $\mathscr{D}\left(f_{1}, \ldots, f_{k}\right)=0$ назьвается $k$-линейнылм уравнением. В случае $k=2$ эта конструкция дает известные билинейные операторы и уравнения Хироты, получившие важные приложения в теории интегрируемых систем. Трилинейные уравнения относятся к введенному в [1] классу многомерных векторных теорем сложения вида

$$
\sum_{\ell} a_{\ell}(u) b_{\ell}(v) c_{\ell}(u+v)=1
$$

Уравнение $\mathscr{D}\left(f_{1}, f_{2}, f_{3}\right)(u, v)=0$ сводится к полиномиальному соотношению между логарифомическими производными функций $f_{1}(u), f_{2}(v)$ и $f_{3}(u+v)$. Здесь мы получим такие соотношения для функций на многообразиях Якоби плоских алгебраических кривых.

Введем оператор

$$
Q(D)\left(f_{1}, f_{2}, f_{3}\right)(u, v)=\frac{\mathscr{D}\left(f_{1}, f_{2}, f_{3}\right)(u, v)}{f_{1}(u) f_{2}(v) f_{3}(u+v)} .
$$

Положим $D_{k}=\partial / \partial z_{k}$, где $z=\left(z_{1}, \ldots, z_{g}\right)$. Пусть $g=1$ и $\rho_{i}(z)=\left(\log f_{i}(z)\right)^{\prime}$. Тогда

$$
\begin{aligned}
& Q\left(D_{1}\right)=\rho_{1}(u)+\rho_{2}(v)-\rho_{3}(u+v), \\
& Q\left(D_{1}^{2}\right)=\left(\rho_{1}(u)+\rho_{2}(v)-\rho_{3}(u+v)\right)^{2}+\rho_{1}^{\prime}(u)+\rho_{2}^{\prime}(v)+\rho_{3}^{\prime}(u+v) .
\end{aligned}
$$

Общее решение функционального уравнения $Q\left(D_{1}\right)=0$ дается линейными функциями $\rho_{i}$, т.е. $f_{i}(z)=\exp \left(\alpha\left(z-\beta_{i}\right)^{2}+\gamma_{i}\right)$, где $\alpha, \beta_{i}$ и $\gamma_{i}$ - постоянные, причем $\beta_{1}+\beta_{2}=\beta_{3}$. Частное решение функционального уравнения $Q\left(D_{1}^{2}\right)=0$ было получено $Г$. Фробениусом и Л. Штикельбергером [2]. Оно было применено к точно решаемшім задачам квантовой механики [3]. Общее решение этого функционалшного уравнения описано в [4]. Функции $f_{i}$ задают волновую функцию основного состояния для квантовой задачи трех тел. В случае общего положения решение представляет собой функции вида $\rho_{i}(z)=\zeta\left(z-\delta_{i}\right)-\beta_{i}$, т.е. $f_{i}(z)=e^{-\beta_{i} z+\gamma_{i}} \sigma\left(z-\delta_{i}\right)$, где $\zeta(z)$ и $\sigma(z)$ - эллиптические функции Вейерштрасса и $\beta_{i}, \gamma_{i}$ и $\delta_{i}$ - постоянные, причем $\beta_{1}+\beta_{2}=\beta_{3}$, $\delta_{1}+\delta_{2}=\delta_{3}$. Отметим еще нормированное частное решение $f_{i}(z)=\frac{1}{\sqrt{2 \pi \Delta}} \exp \left(-\frac{\left(z-q_{i}\right)^{2}}{2 \Delta^{2}}\right)$, где $q_{i}$ - постоянные и $\Delta=\left(q_{1}+q_{2}-q_{3}\right) / \sqrt{3}$.

Теория трилинейных уравнений для целшхх функций $f_{i}(z), i=1,2,3$, опирается на следующие резултаты.

Лемма 1. Пусть для некоторого $N$ имеет место разложсение

$$
f_{1}(u+z) f_{2}(v+z) f_{3}((u+v)-z)=\sum_{k=1}^{N} \varphi_{k}(u, v) \psi_{k}(z) .
$$

Тогда для любого набора линейных дифференциальных операторов $L_{1}, \ldots, L_{N+1}$ существует нетривиальный набор констант $c_{1}, \ldots, c_{N+1}$ такой, что $Q(D)\left(f_{1}, f_{2}, f_{3}\right)=0$ для onepamopa $D=\sum_{i=1}^{N+1} c_{i} L_{i}$.

Для $g$-мерных тета-функций первого порядка и родственных им функций разложение из леммы 1 сушествует при $N=3^{g}$. Например, если $f_{1}=f_{2}=f_{3}=\sigma$, то для $g=1$ имеем $\left(\psi_{1}, \psi_{2}, \psi_{3}\right)=\sigma^{3}\left(1, \wp, \wp^{\prime}\right)$, а для $g=2$ имеем $\left(\psi_{1}, \ldots, \psi_{9}\right)=\sigma^{3}\left(1, \wp_{i, j}, \wp_{i, j, k}, \wp_{2,2} \wp_{1,1}-\wp_{1,2}^{2}\right)$,

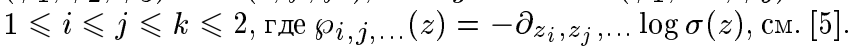


ЛЕмма 2. Пусть $\phi: \mathbb{C} \rightarrow \mathbb{C}^{g}$ - некоторое гладжое, регулярное при $\xi=0$ отображсение u $G(\xi)$ - мероморфная функиия в окрестности $\xi=0$. Образуем функцию

$$
F(u, v, \xi)=G(\xi) f_{1}(u+\phi(\xi)) f_{2}(v+\phi(\xi)) f_{3}((u+v)-\phi(\xi)) .
$$

Тогда, если в разложении $F(u, v, \xi)$ по степеням $\xi$ коэффициент при $\xi^{k}$ для некоторого $k$ равен нулю, то $Q(D)\left(f_{1}, f_{2}, f_{3}\right)=0$ для соответствующего оператора $D$.

Следующий результат - ключевой для приложений леммы 2.

Теорема 3. Пусть б-сигма-функция, ассоциированная с плоской алгебраической кривой

$$
V=\left\{(x, y) \in \mathbb{C}^{2} \mid y^{n}-x^{s}-\sum_{n s-i n-j s>0} \lambda_{n s-i n-j s} x^{i} y^{j}=0\right\}, \quad \text { где } \quad(n, s)=1 .
$$

Тогда имеет место формула

$$
\sigma(u) \sigma(v) \sigma(u+v) R_{3 g}(x, y)=c \psi^{-3}(x, y) \sigma(u+A(x, y)) \sigma(v+A(x, y)) \sigma(u+v-A(x, y)),
$$

где $R_{3 g}(x, y)$ - целая функция на $V$, задающая закон сложения на $\mathrm{Sym}^{g}(V)$, см. [6], $c-$ константа, $A: V \rightarrow \mathrm{Sym}^{g}(V)$ - отображсение Абеля и

$$
\psi(x, y)=\exp \left\{-\int_{\infty}^{(x, y)}\left\langle A^{*}\left(x^{\prime}, y^{\prime}\right), \mathrm{d} A\left(x^{\prime}, y^{\prime}\right)\right\rangle\right\}
$$

Здесь $A^{*}$ - ассоциированное с $A$ отображение, задаваемое абелевыми интегралами второго рода.

Лемма 2 и теорема 3 в сочетании с теоремой Вейерштрасса о пробелах приводят к теоремам сложения, характеризующим сигма-функции на якобианах.

Теорема 4. Наименьший порядок линейного дифференциального оператора $D$ такого, что $Q(D)(\sigma, \sigma, \sigma)(u, v)=0$, равен $g+1$, где $g=(n-1)(s-1) / 2-$ род кривой $V$.

Например, при $(n, s)=(2,3)$ имеем: $g=1$ и оператор наименьшего порядка $D=D_{1}^{2}$; при $(n, s)=(2,5)$ имеем: $g=2$ и, соответственно, $D=2 D_{1}+D_{2}^{3}$. Положим $\rho_{i}^{(j, k)}(z)=$ $\partial^{j+k} \log f_{i}(z) / \partial z_{1}^{j} \partial z_{2}^{k}$. Тогда

$$
\begin{gathered}
Q\left(2 D_{1}+D_{2}^{3}\right)=2 r_{(1,0)}+r_{(0,3)}+3 r_{(0,2)} r_{(0,1)}+r_{(0,1)}^{3}, \\
\text { где } r_{(j, k)}=\rho_{1}^{(j, k)}(u)+\rho_{2}^{(j, k)}(v)+(-1)^{j+k} \rho_{3}^{(j, k)}(u+v) .
\end{gathered}
$$

\section{СПИСОК ЛИТЕРАТУРЫ}

[1] V.M. Buchstaber, I. M. Krichever // Internat. Math. Res. Notices. 1996. V. 10. P. 505-513. [4] V. M. Buchstaber, A. M. Perelomov // Amer. Math. Soc. Transl. Ser. 2. 1996. V. 175. P. 15-34. [6] V. M. Buchstaber, D. V. Leykin // J. Nonlinear Math. Ohys. 2005. V. 12. Suppl. 1. P. 106-123. [5] V. M. Buchstaber, V.Z. Enolskii, D. V. Leykin // Rev. Math. Math. Phys. 1997. V. 10. № 2. P. 3-120. [3] F. Calogero // Lett. Nuovo Cimento. 1976. V. 16. P. 77-80. [2] G. Frobenius, L. Stickelberger // J. Reine Angew. Math. 1880. V. 88. P. $146-184$.

Математический институт им. В. А. Стеклова РАН; Институт магнетизма, Киев, Украина E-mail: buchstab@mendeleevo.ru; dile@imag.kiev.ua
Представлено А. В. Булинским Принято редколлегией 11.02.2004 